\title{
Molten salt eutectics from atomistic simulations
}

\author{
Saivenkataraman Jayaraman, ${ }^{*}$ Aidan P. Thompson, and O. Anatole von Lilienfeld ${ }^{\dagger}$ \\ Sandia National Laboratories, Albuquerque, New Mexico 87185, USA \\ (Received 1 November 2010; revised manuscript received 10 May 2011; published 1 September 2011)
}

\begin{abstract}
Despite their importance for solar thermal power applications, phase-diagrams of molten salt mixture heat transfer fluids (HTFs) are not readily accessible from first principles. We present a molecular dynamics scheme general enough to identify eutectics of any HTF candidate mixture. The eutectic mixture and temperature are located using the liquid mixture free energy and the pure component solid-liquid free energy differences. The liquid mixture free energy is obtained using thermodynamic integration over particle identity transmutations sampled with molecular dynamics at a single temperature. Drawbacks of conventional phase diagram mapping methodologies are avoided by not considering solid mixtures, thereby evading expensive computations of solid phase free energies. Numerical results for binary and ternary mixtures of alkali nitrates agree well with experimental measurements.
\end{abstract}

DOI: 10.1103/PhysRevE.84.030201

PACS number(s): 64.70.D-, 61.66.Fn, 64.75.Cd, 81.30.Dz

According to Carnot's cycle, the maximum efficiency of solar thermal power facilities increases with the temperature difference between melting and thermal decomposition of employed heat transfer fluids (HTFs). Consequently, new formulations of low melting but temperature resistant molten salt mixtures are actively sought [1]. Competitive formulations currently include eutectics of alkali and alkali-metal earth nitrates and nitrite mixtures. Unfortunately, the dimensionality of the combinatorial multicomponent space of all possible cations and anions prohibits exhaustive scanning using computer simulation. Once an optimal HTF formulation is identified, however, its realization through simple mixing is readily accomplished in experiment. Identifying new HTFs therefore represents a rewarding challenge for atomistic first principles materials design efforts using theory and computation. $A b$ initio (AI) based design, through an efficient combination of first principles methods and optimization algorithms [2], has already been applied to inverting band structures [3], identifying stable alloys [4], designing heterogeneous catalysts [5,6], or discovering ternary metal oxides for batteries [7]. Identity transformations, also called "alchemical" transmutations [8], have been successfully applied to compute compositions in the earth's core [9], phase stability in solid solutions [10], and a wide variety of biochemical applications [11], among others.

The Gibbs criteria for phase equilibrium dictates that temperature, pressure, and chemical potential of individual components must be equal in coexisting phases. Therefore, knowledge not only of average liquid configurations but also of the solid mixture's structure is essential. For phase transitions of mixtures, the problem is hence strongly linked to the challenge of predicting molecular crystals from first principles [12], which has been addressed for co-crystals only recently $[7,13]$. Recent efforts to compute solid-liquid phase equilibria of hard sphere mixtures [14], Lennard-Jones mixtures [15], alloys [16], or solid and liquid mixtures of the $\mathrm{Rb} / \mathrm{Cs}$ fluoride binary [17] demonstrate the intricacies involved

\footnotetext{
*sjayara@sandia.gov

${ }^{\dagger}$ Current address: Argonne Leadership Computing Facility, Argonne National Laboratory, Argonne, Illinois 60439, USA.
}

in mapping out entire phase diagrams. For the identification of HTF mixtures with minimal melting points, however, it is sufficient to simply focus on eutectics, rather than having to screen the entire solid-liquid phase diagram. Here, we present a first principles approach for directly estimating eutectic compositions and temperatures for any number of components, relying on a combination of only a couple of molecular dynamics (MD) calculations per mole fraction at a single temperature and knowledge about only the pure solid - and not the solid mixture. While our scheme could be applied to any HTF candidate using electronic-structure-based $a b$ initio molecular dynamics (AIMD) [18], here we illustrate it without any loss of generality for classical interatomic potential models. Our protocol involves a series of short MD calculations along "alchemical" transmutations that turn one chemical component into another, permitting the calculation of excess free energies of mixing for the liquid as a function of composition. The third Gibbs criterion for the eutectic can be represented graphically by a common tangent plane drawn between the free energy surfaces of coexisting phases [19]. Assuming the "simple eutectic;" namely, eutectic solid phases well approximated by pure solids, the Gibbs criterion reduces to the tangent between the liquid free energy of mixing and the linear hyperplane connecting the solid-liquid free energy differences of the pure components. Such an approximation eliminates the necessity to conduct computationally challenging particle identity swaps in the solid phase.

Numerical evidence for the presented scheme is produced and compared to experimental data of eutectics involving binary and ternary mixtures of lithium, sodium, and potassium nitrate. The choice of these molten salts for assessing the performance of our approach is due to the fact that (a) they already constitute significant parts of currently used HTF formulations [1], (b) direct comparison is possible with experimental and theoretical literature, and (c) their monovalency permits straightforward identity interconversion. Nonetheless, our method is versatile enough for effortless extension to any system of interest. We find our results to be in good agreement with experimentally determined values [20-23]. Since we focus on mixtures of lithium, sodium, and potassium nitrates, henceforth they will be referred to as $\mathrm{Li} / \mathrm{Na}$, $\mathrm{Li} / \mathrm{K}, \mathrm{Na} / \mathrm{K}$, and $\mathrm{Li} / \mathrm{Na} / \mathrm{K}$, respectively. 
For a binary liquid mixture with $N_{\mathrm{A}}$ and $N_{\mathrm{B}}$ particles of component $\mathrm{A}$ and $\mathrm{B}$, respectively, the free energy of mixing as a function of mole fraction $x_{\mathrm{A}}$ can be written as

$$
g_{\ell}^{\text {mix }}\left(x_{\mathrm{A}}\right)=g_{\ell}\left(x_{\mathrm{A}}\right)-x_{\mathrm{A}} g_{\mathrm{A} \ell}^{\circ}-x_{\mathrm{B}} g_{\mathrm{B} \ell}^{\circ}=g^{\mathrm{id}}\left(x_{\mathrm{A}}\right)+g_{\ell}^{\mathrm{ex}}\left(x_{\mathrm{A}}\right),
$$

where $g_{\ell}$ is the total molar free energy of the liquid mixture, and $g_{\mathrm{A} \ell}^{\circ}$ and $g_{\mathrm{B} \ell}^{\circ}$ are the free energies of liquid pure components $\mathrm{A}$ and $\mathrm{B}$, respectively. $g_{\ell}^{\mathrm{ex}}$ is the excess free energy of mixing for the liquid phase, and $g^{\text {id }}=k_{B} T\left(x_{\mathrm{A}} \ln x_{\mathrm{A}}+x_{\mathrm{B}} \ln x_{\mathrm{B}}\right)$ is the ideal free energy of mixing ( $k_{B}$ being the Boltzmann constant).

We compute $g_{\ell}^{\text {ex }}$ via $\Delta \mu_{\mathrm{AB}}$, which is the free energy change associated with an identity transformation of a particle of $\mathrm{B}$ into a particle of A. At composition $x_{\mathrm{A}}(\lambda=0)=N_{\mathrm{A}} /\left(N_{\mathrm{A}}+\right.$ $N_{\mathrm{B}}$ ), for example, a particle B can be reversibly transformed into $\mathrm{A}$ as a continuous function of an order parameter $0 \leqslant$ $\lambda \leqslant 1$, resulting in $x_{\mathrm{A}}(\lambda=1)=\left(N_{\mathrm{A}}+1\right) /\left(N_{\mathrm{A}}+N_{\mathrm{B}}\right)$. Figure 1 schematically illustrates such an identity transformation. We assume a linear function in $\lambda$ for the potential, $u_{i j}$, of a randomly picked transforming particle $j$ interacting with any other particle $i$ of the mixture at $x_{\mathrm{A}}: u_{i j}(\lambda)=$ $\lambda u_{i \mathrm{~A}}+(1-\lambda) u_{i \mathrm{~B}}$. The associated change in free energy can be evaluated via thermodynamic integration [24]:

$$
\Delta \mu_{\mathrm{AB}}=\int_{0}^{1} d \lambda\left\langle\frac{\partial U}{\partial \lambda}\right\rangle_{\lambda}=\int_{0}^{1} d \lambda\left\langle\sum_{i}\left(u_{i \mathrm{~A}}-u_{i \mathrm{~B}}\right)\right\rangle_{\lambda},
$$

where $U=\sum_{i, j} u_{i j}$ is the total potential energy of the system.

As such, $\Delta \mu_{\mathrm{AB}}$ represents a statistical mechanical finite difference version of a "transmutating" or "alchemical" potential, as also used within the isomolar semigrand ensemble theory [25] or its electronic structure analog [26].

For a binary liquid, $g_{\ell}^{\text {ex }}$ is then computed from $\Delta \mu_{\mathrm{AB}}$ using

$$
g_{\ell}^{\mathrm{ex}}\left(x_{\mathrm{A}}\right)=\int_{0}^{x_{\mathrm{A}}} d x_{\mathrm{A}}^{\prime} \Delta \mu_{\mathrm{AB}}\left(x_{\mathrm{A}}^{\prime}\right)-x_{\mathrm{A}} \int_{0}^{1} d x_{\mathrm{A}}^{\prime} \Delta \mu_{\mathrm{AB}}\left(x_{\mathrm{A}}^{\prime}\right),
$$

where $1=x_{\mathrm{A}}+x_{\mathrm{B}}$ and where for $x_{\mathrm{A}}=0,1$ (i.e., for the pure components), $g_{\ell}^{\mathrm{ex}}=0$. Extension to the ternary liquid mixture $\mathrm{A} / \mathrm{B} / \mathrm{C}$ yields

$$
\begin{aligned}
g_{\ell}^{\mathrm{ex}}\left(x_{\mathrm{A}}, x_{\mathrm{C}}\right)= & \int_{0}^{x_{\mathrm{A}}} d x_{\mathrm{A}}^{\prime} \Delta \mu_{\mathrm{AB}}\left(x_{\mathrm{A}}^{\prime}, x_{\mathrm{C}}\right) \\
& -\frac{x_{\mathrm{A}}}{x_{\mathrm{A}}+x_{\mathrm{B}}} \int_{0}^{x_{\mathrm{A}}+x_{\mathrm{B}}} d x_{\mathrm{A}}^{\prime} \Delta \mu_{\mathrm{AB}}\left(x_{\mathrm{A}}^{\prime}, x_{\mathrm{C}}\right) \\
& +\frac{x_{\mathrm{A}}}{x_{\mathrm{A}}+x_{\mathrm{B}}} g_{\mathrm{AC} \ell}^{\mathrm{ex}}\left(x_{\mathrm{C}}\right)+\frac{x_{\mathrm{B}}}{x_{\mathrm{A}}+x_{\mathrm{B}}} g_{\mathrm{BC} \ell}^{\mathrm{ex}}\left(x_{\mathrm{C}}\right),
\end{aligned}
$$

where $1=x_{\mathrm{B}}+x_{\mathrm{A}}+x_{\mathrm{C}}, \Delta \mu_{A B}$ is the change in free energy due to the identity transformation of $\mathrm{B}$ to $\mathrm{A}$ at $\left(x_{A}, x_{C}\right)$, and $g_{\mathrm{AC} \ell}^{\mathrm{ex}}$ and $g_{\mathrm{BC} \ell}^{\mathrm{ex}}$ are the respective binary excess free energies. Setting $x_{\mathrm{C}}=0$ in Eq. (4) yields Eq. (3). Derivations of these relations will be presented elsewhere. For rapid numerical detection of tangent temperatures and locations in the ternary, a fifthorder polynomial was fit to $g_{\ell}^{\text {mix }}$, yielding minimal least square deviation at, and in the vicinity of, the minimum.

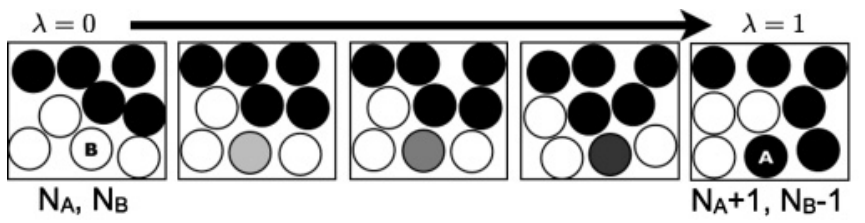

FIG. 1. Schematic of identity transformation method in a binary mixture of A and B. A particle of component B (white) is transformed to component A (black) via a sequence of transforming particles (gray).

For the eutectic solid phase, we make the approximation that the free energy and composition of each solid phase is equal to that of the corresponding pure component solid phase. As a result, the Gibbs phase equilibrium conditions rigorously imply the following necessary condition: At the eutectic temperature the hyperplane connecting the pure component solid-liquid free energy differences, $g_{s \ell}^{\circ}$, is a tangent to the liquid free energy of mixing surface, $g_{\ell}^{\text {mix }}$. The point of tangency gives the liquid composition. The solid-liquid connecting hyperplane consists of a line for the binary and of a plane for the ternary eutectic. It can be written as $g_{s \ell}^{\circ}=\sum_{i} x_{i} \Delta_{s \ell} g_{i}^{\circ}$, where $\Delta_{s \ell} g_{i}^{\circ}=g_{i s}^{\circ}-g_{i \ell}^{\circ}$ is the difference in free energies of the pure solid and liquid phases. These can be calculated as a function of temperature using a variety of established methods [27]. Here, the temperature dependence of $g_{\ell}^{\text {mix }}$ is obtained from $g^{\text {id }}$ only, assuming $g_{\ell}^{\text {ex }}$ to be temperature invariant within the investigated temperature range.

Using LAMMPS [28,29], $\Delta \mu_{\mathrm{AB}}$ in Eq. (2) was computed through numerical integration over 11 equidistant $\lambda$ values at 13 discrete compositions for the binary mixtures and at 45 discrete compositions for the $\mathrm{Li} / \mathrm{Na} / \mathrm{K}$ mixture equally spaced over the entire composition range. For each composition, a single transforming particle was chosen at random from the bulk liquid. The dependence of $\Delta \mu_{\mathrm{AB}}$ on the choice of particle was found to be within statistical uncertainties, which were computed using the error estimation procedure described in Ref. [30].

Inset (a) in Fig. 2 illustrates the calculation of $\Delta \mu_{\mathrm{AB}}$ in Eq. (2) for the integrand as a function of $\lambda$ at $x_{\mathrm{Li}}=0.5$ in the $\mathrm{Li} / \mathrm{K}$ mixture. The well-behaved function indicates that, for less than $11 \mathrm{MD}$ runs, reasonable results would have been obtained. Inset (b) of Fig. 2 also features cation-anion radial distribution functions (RDF) for 4 mole fractions of the $\mathrm{Li} / \mathrm{K}$ liquid. The changes in height and location of the peaks suggest nonuniform structural changes in the first and second solvation shells as a function of mixing. Such subtle structural changes play an important role in phase equilibria [31] and are fully accounted for in our calculations of $g_{\ell}^{\text {mix }}$. Figure 2 illustrates the tangent-based location for the Li/K binary eutectic. As the temperature increases, the increase in entropy causes the connecting line $g_{s \ell}^{\circ}$ to shift upward and increase in slope while the liquid free energy of mixing curve deepens. The temperature at which the two are tangent gives the predicted eutectic temperature, (i.e., $423 \mathrm{~K}$; experiment gives $410 \mathrm{~K}$ [22]) and the corresponding mole fraction (indicated by the pink arrow) $x_{\mathrm{Li}}=0.59$ (experiment gives 0.55 [22]). Analogous trends were observed for the $\mathrm{Li} / \mathrm{Na}$ and $\mathrm{Na} / \mathrm{K}$ binaries (not shown). 


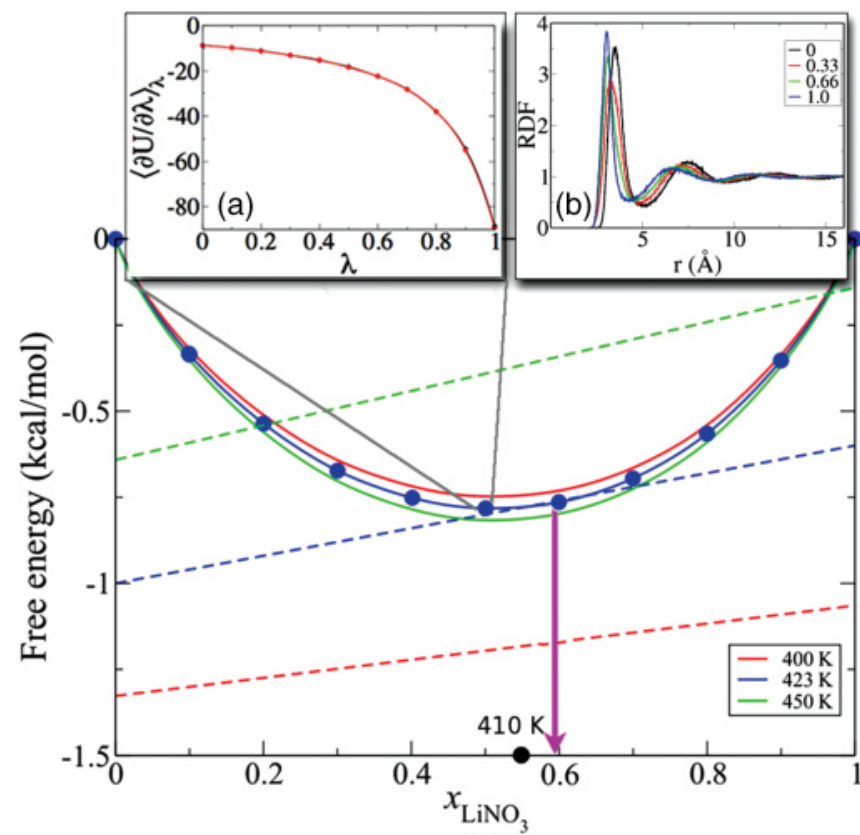

FIG. 2. (Color) Calculated free energies for the $\mathrm{Li} / \mathrm{K}$ nitrate binary at three temperatures. Smooth lines indicate the free energies of mixing for the liquid phase $\left[g_{\ell}^{\operatorname{mix}}\left(x_{\mathrm{Li}}\right)\right]$ according to Eqs. (1) and (3). Blue filled circles show the compositions at which $\Delta \mu_{\mathrm{AB}}$ were computed. The statistical uncertainties in free energies were smaller than the size of the blue symbols and are not shown here. Dashed lines connect pure solid-liquid free energy differences $\left(g_{s \ell}^{\circ}\right)$. Black filled circle is experimental $\mathrm{Li} / \mathrm{K}$ eutectic [22]. Insets: (a) Integrand for thermodynamic integration in Eq. (2), $\langle\partial U / \partial \lambda\rangle$ for $\mathrm{Li} / \mathrm{K}$ system at $x_{\mathrm{Li}}(\lambda=0)=0.5 \mapsto x_{\mathrm{Li}}(\lambda=1)=0.5017$. (b) Liquid phase cation-anion radial distribution function for the $\mathrm{Li} / \mathrm{K}$ system at $x_{\mathrm{Li}}=0$ (black), 0.33 (red), 0.66 (green), and 1.0 (blue).

The corresponding extension to the ternary mixture is displayed in insets (a)-(c) of Fig. 3. With increasing temperature, the connecting plane $g_{s \ell}^{\circ}$ rises while the ternary free energy surface of mixing drops. The temperature at which the two surfaces touch gives the predicted eutectic temperature, and the corresponding mole fraction is indicated by the pink arrow [inset (b) of Fig. 3]. Figure 3 also compares the computed ternary eutectic to the experimental eutectic. The three branches emanating from the ternary eutectic represent binary eutectics in the presence of the third component. Crosses on these branches indicate temperature contours. The branches were obtained as the point of tangency of planes containing connecting lines $g_{s \ell}^{\circ}$ for the three binary pairs $\mathrm{Li} / \mathrm{Na}$, $\mathrm{Li} / \mathrm{K}$, and $\mathrm{Na} / \mathrm{K}$ with the ternary $g_{\ell}^{\mathrm{mix}}$, evaluated at temperatures ramped up from the ternary eutectic to the respective binary eutectic points. The mismatch of the branch endpoints with respect to the values calculated for the pure binaries is not surprising, since all ternary tangents were identified using the aforementioned polynomial fit to $g_{\ell}^{\mathrm{mix}}$ that deviates the least at the center of the ternary diagram and the most at its binary boundaries.

The overall agreement with experiment is good; the deviation of calculated compositions from most recent experimental values and the variance among experimental eutectic compositions are of similar magnitude. Calculated

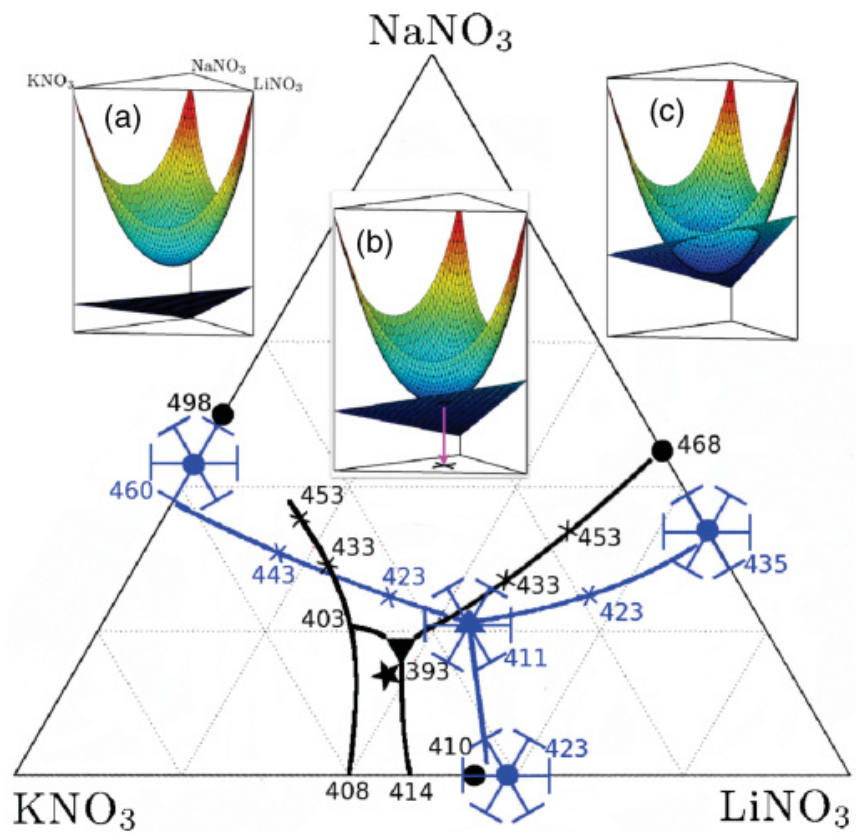

FIG. 3. (Color) Calculated (blue) and experimental (black) eutectics for the $\mathrm{Li} / \mathrm{Na} / \mathrm{K}$ nitrate ternary. The blue lines represent binary eutectics in the presence of the third component and are not meant to depict the ternary phase diagram. The black triangle, black curves [20], and black pentagram [21] are ternary data, while the filled black circles are the binary eutectics from Refs. [22,23]. Crosses indicate temperature contours $(\mathrm{K})$. Insets show the free energy of mixing for the liquid phase $\left(g_{\ell}^{\text {mix }}\right)$ and pure solid-liquid free energy difference connecting plane $\left(g_{s \ell}^{\circ}\right)$ at three temperatures: $T_{(\mathrm{a})}<T_{(\mathrm{b})}<T_{(\mathrm{c})}$.

eutectic temperatures, however, can deviate by up to tens of kelvin. These differences are likely due to shortcomings in the employed interatomic potential. The binaries are experimentally observed to be simple eutectics; however, there is disagreement among experimental studies regarding the presence of intermediate solid phases for the $\mathrm{LiNO}_{3}-\mathrm{KNO}_{3}$ mixture [20,22]. Although experimental data indicates that the ternary is not a simple eutectic, our calculated eutectic point is in good agreement with the experimental observation, as shown in Fig. 3. The simple eutectic approximation yields reasonable estimates of the eutectic point, even for systems which are not simple eutectics.

While results for alternative thermodynamic modeling methods achieve better agreement with experiment, they are based on significant empirical input. In contrast, all our calculations require no experimental data, being based solely on atomistic MD using empirical interatomic potential models. This can be a crucial difference for exploring novel mixtures or unusual conditions for which no experimental data is available. Furthermore, in the absence of accurate interatomic potentials it would be straightforward to use AIMD within the presented scheme. Alchemical changes within AIMD can be performed in a manner analogous to that described here [32,33]. The corresponding anticipated increase in computational cost could be mitigated by reducing the necessary number of mole fractions or through coarser integration of Eq. (2) by virtue of linearizing schemes for the integrand, $\langle\partial U / \partial \lambda\rangle_{\lambda}$ [inset (a) in Fig. 2)], as recently proposed by one of us [34]. 
Using identity transmutations, the free energies of liquid mixtures were computed as a function of mole fraction from atomistic simulations. The point of tangency between the hyperplane connecting the pure component solid-liquid free energy differences $\left(g_{s \ell}^{\circ}\right)$ and a polynomial fit to the liquid free energy of mixing surface $\left(g_{\ell}^{\mathrm{mix}}\right)$ allows reliable prediction of the eutectic temperature and liquid composition. We have numerically demonstrated the method for all the binaries and the ternary of $\mathrm{Li} / \mathrm{Na} / \mathrm{K}$ nitrate mixtures. Our computed eutectics compare favorably to existing experimental measurements. The two main ingredients for this approach are (a) the free energy difference between the pure component solid and liquid phases (from theory or experiment) and (b) the liquid phase free energy of mixing, both as a function of temperature. Future work will deal with extension of the method to mixtures of divalent, multivalent, or molecular ions.

\section{ACKNOWLEDGMENTS}

OAvL acknowledges support from SNL's Laboratory Directed Research \& Development Truman program, No. 120209. Sandia National Laboratories is a multiprogram laboratory operated by Sandia Corporation, a wholly owned subsidiary of Lockheed Martin Corporation, for the US Department of Energy's National Nuclear Security Administration under contract DE-AC04-94AL85000.
[1] R. W. Bradshaw and N. P. Siegel, in ES2008, Proceedings of the ASME 2rd International Conference on Energy Sustainability (ASME, New York, 2009), Vol. 2, pp. 631-637.

[2] J. Hafner, C. Wolverton, G. Ceder, and G. Editors, MRS Bulletin 31, 659 (2006).

[3] A. Franceschetti and A. Zunger, Nature (London) 402, 60 (1999).

[4] G. H. Jóhannesson, T. Bligaard, A. V. Ruban, H. L. Skriver, K. W. Jacobsen, and J. K. Nørskov, Phys. Rev. Lett. 88, 255506 (2002).

[5] J. K. Nørskov, T. Bligaard, J. Rossmeisl, and C. H. Christensen, Nature Chemistry 1, 37 (2009).

[6] D. Sheppard, G. Henkelman, and O. A. von Lilienfeld, J. Chem. Phys. 133, 084104 (2010).

[7] G. Hautier, C. C. Fischer, A. Jain, T. Mueller, and G. Ceder, Chem. Mater. 22, 3762 (2010).

[8] T. P. Straatsma and J. A. McCammon, Annu. Rev. Phys. Chem. 43, 407 (1992).

[9] D. Alfè, M. J. Gillan, and G. D. Price, Nature (London) 405, 172 (2000).

[10] N. Marzari, S. deGironcoli, and S. Baroni, Phys. Rev. Lett. 72, 4001 (1994).

[11] C. D. Christ, A. E. Mark, and W. F. van Gunsteren, J. Comput. Chem. 31, 1569 (2010).

[12] J. Maddox, Nature (London) 335, 201 (1988).

[13] A. R. Oganov, Y. Ma, A. O. Lyakhov, M. Valle, and C. Gatti, Rev. Mineral. Geochem. 71, 271 (2010).

[14] W. G. T. Kranendonk and D. Frenkel, Mol. Phys. 72, 679 (1991).

[15] M. R. Hitchcock and C. K. Hall, J. Chem. Phys. 110, 11433 (1999).

[16] M. Asta, J. J. Hoyt, and A. Karma, Phys. Rev. B 66, 100101 (2002).
[17] O. Beneš, P. Zeller, M. Salanne, and R. J. M. Konings, J. Chem. Phys. 130, 134716 (2009).

[18] R. Iftimie, P. Minary, and M. E. Tuckerman, Proc. Nat. Acad. Sci. USA 102, 6654 (2005).

[19] C. H. P. Lupis, Chemical Thermodynamics of Materials (North Holland, Amsterdam, 1983).

[20] A. G. Bergman and K. Nogoev, Zh. Neorg. Khim. 9, 1423 (1964).

[21] H. R. Carveth, J. Phys. Chem. 2, 209 (1898).

[22] M. J. Maeso and J. Largo, Thermochim. Acta 223, 145 (1993).

[23] C. M. Kramer and C. J. Wilson, Thermochim. Acta 42, 253 (1980).

[24] J. G. Kirkwood, J. Chem. Phys. 3, 300 (1935).

[25] T. I. Morrow and E. J. Maginn, J. Chem. Phys. 122, 054504 (2005).

[26] O. A. von Lilienfeld and M. E. Tuckerman, J. Chem. Phys. 125, 154104 (2006).

[27] S. Jayaraman, A. P. Thompson, O. A. von Lilienfeld, and E. J. Maginn, Ind. Eng. Chem. Res. 49, 559 (2010).

[28] S. Plimpton, J. Comput. Phys. 117, 1 (1995).

[29] See Supplemental Material at http://link.aps.org/supplemental/ 10.1103/PhysRevE.84.030201 for details of the simulation setup and the intermolecular potential.

[30] S. Jayaraman and E. J. Maginn, J. Chem. Phys. 127, 214504 (2007).

[31] A. R. Denton, G. Kahl, and J. Hafner, J. Non-Cryst. Solids 250, 15 (1999).

[32] O. A. von Lilienfeld and M. E. Tuckerman, J. Chem. Theory Comput. 3, 1083 (2007).

[33] K. Leung, S. B. Rempe, and O. A. von Lilienfeld, J. Chem. Phys. 130, 204507 (2009).

[34] O. A. von Lilienfeld, J. Chem. Phys. 131, 164102 (2009). 